Proceedings

\title{
Information Ecology and Cognitive Justice: Core Value and Methodological Principles of Information Ecology ${ }^{\dagger}$
}

\author{
Kang Ouyang \\ Huazhong University of Science and Technology, Wuhan 430074, China; kouyang@mail.hust.edu.cn; \\ Tel.: +86-27-8779-3020; Fax: +86-27-8754-5438 \\ + Presented at the IS4SI 2017 Summit DIGITALISATION FOR A SUSTAINABLE SOCIETY, Gothenburg, \\ Sweden, 12-16 June 2017.
}

Published: 9 June 2017

\section{The Information Ecology and Its Significance}

From the perspective of science, the introduction of information ecology is the expansion and application of the principles and methods of ecology in the information science researches.

From the perspective of real practice, the real problems that the information ecology encounters is the irrationality and injustice in the information survival, information processing, information transit and information sharing, including the information overload, information pollution, information harassment and information crime. It calls for the fairness as well as justice in theinformation occupation which provides the necessary requisite for the cognitive justice.

If Chinese researches on the information science generally lag behind western developed countries, the emergence of the information ecology in modern China which has been attached great importance to represents an advanced self-awareness. Information enables Chinese information science to rise to the similar standard of international information circle and it can realize the advanced development via taking the advantage of the Chinese market-the world's largest information system and the fastest expanding information market, which would lead the world's information technology and the future of the culture in a step-by-step manner.

\section{Core Values of the Information Ecology}

Cognitive justice is the necessary basis and the cognitive prerequisite for social justice and fairness. However the information justice serves as the prerequisite for the cognitive justice. Information Ecology promotes the information justice and cognitive justice through the focus and researches on the information ecology and it is endowed with unique core values.

On Information and Information Researches, Professor Zhong Yixin depicts the process of information ecology: ontological information $\rightarrow$ epistemological information $\rightarrow$ knowledge $\rightarrow$ intelligent strategies $\rightarrow$ intelligent behavior, which enlightens us a lot.

(1) Whether the external social and political cultural environment is rational and orderly, whether it is harmonious, and whether there exist external constraints which hinder the natural existence and healthy evolution in the information ecology etc.

(2) Whether in the certain information system the plural subjects, complex objects and the intermediary elements are sound, the organization healthy, the structure reasonable and the function comprehensive, etc.

(3) Whether in there certain information system the operation approach is reasonable and orderly, the operation process steady and safe, the operation monitoring timely and reliable and the operation function effective, etc. 
(4) Whether in the specific information system the trend and direction incertain information system is reasonable and healthy, the transition method suits the logic and the how to better the structure for the future form in a more reasonable manner, etc.

\section{Methodological Principles in Information Ecology}

Methodology in information ecology is of great importance to the research and application of information ecology which should be attached great importance to.

Professor Zhong Yixin offers the logic chain and advocates the integration research of information society $\rightarrow$ information education $\rightarrow$ information philosophy $\rightarrow$ information science $\rightarrow$ information project $\rightarrow$ information management $\rightarrow$ information economy $\rightarrow$ information society which is of great instructive significance.

We put forward several methodological principles of researches on information ecology from the perspective of cognitive justice in hope that we can direct the value orientation of the information ecology.

First, objectivity. The original properties, features and regularities are respected which correspond to principles and methods of the information ecology system to study problems of the research information ecology and enhance the publicity, scientificness and transparency of the information system.

Second, systematicity. Status of various kinds of information elements in the information ecological system should be respected and valued and meanwhile the systematic features and systematic functions of information ecology should be strengthened and therefore the integrity, coordination and organicity of the information ecological system can be enhanced.

Third, subjectivity. It is suggested that the dominant positions of different people in the ecological information system be respected and given the great attention, that people's responsibility for the information ecology be implemented and that the purposiveness, value and orientation of the information ecology be further enhanced.

Fourth, the justice. It is suggested that power of different sectors be respected, interests of various parties be secured and the legitimacy, readability and efficiency of the interpretations on the information form be strengthened.

(C) 2017 by the author. Licensee MDPI, Basel, Switzerland. This article is an open access article distributed under the terms and conditions of the Creative Commons Attribution (CC BY) license (http://creativecommons.org/licenses/by/4.0/). 